\title{
Eupatilin induces human renal cancer cell apoptosis via ROS-mediated MAPK and PI3K/AKT signaling pathways
}

\author{
WEI-FENG ZHONG ${ }^{1 *}$, XIAO-HONG WANG ${ }^{2 *}$, BIN PAN $^{3}$, FENG LI $^{4}$, LU KUANG $^{5}$ and ZE-XUAN SU ${ }^{3}$ \\ ${ }^{1}$ Department of Urology, Meizhou People's Hospital, Meizhou, Guangdong 514031; \\ ${ }^{2}$ Department of Nephrology, The Third Affiliated Hospital of Southern Medical University; ${ }^{3}$ Department of Urology, \\ The First Affiliated Hospital of Jinan University, Guangzhou, Guangdong 510630; ${ }^{4}$ Department of Urology, \\ The Fourth Affiliated Hospital of Guangzhou Medical University, Guangzhou, Guangdong 511447; ${ }^{5}$ Department of Urology, \\ The Third Affiliated Hospital of Southern Medical University, Guangzhou, Guangdong 510630, P.R. China
}

Received July 13, 2015; Accepted May 16, 2016

DOI: $10.3892 / \mathrm{ol} .2016 .4989$

\begin{abstract}
Phosphatidylinositol 3-kinase (PI3K)/AKT and mitogen activated protein kinase (MAPK) signaling cascades have significant roles in cell proliferation, survival, angiogenesis and metastasis of tumor cells. Eupatilin, one of the major compounds present in Artemisia species, has been demonstrated to have antitumor properties. However, the effect of eupatilin in renal cell carcinoma (RCC) remains to be elucidated. Therefore, the present study investigated the biological effects and mechanisms of eupatilin in RCC cell apoptosis. The results of the present study demonstrated that eupatilin significantly induced cell apoptosis and enhanced the production of reactive oxygen species (ROS) in 786-O cells. In addition, eupatilin induced phosphorylation of p38 $\alpha$ (Thr180/Tyr182), extracellular signal-regulated kinase 1/2 and c-Jun N-terminal kinase 1/2 (Thr183/Tyr185), and decreased the phosphorylation of PI3K and AKT in 786-O cells in a concentration-dependent manner. Furthermore, the ROS inhibitor N-acetyl-L-cysteine was able to rescue the MAPK activation and $\mathrm{PI} 3 \mathrm{~K} / \mathrm{AKT}$ inhibition induced by eupatilin. Taken together, the results of the present study provide evidence that inhibition of eupatilin induces apoptosis in human RCC via ROS-mediated activation of the MAPK signaling pathway and inhibition of the PI3K/AKT signaling pathway. Thus, eupatilin may serve as a potential therapeutic agent for the treatment of human RCC.
\end{abstract}

Correspondence to: Dr Ze-Xuan Su, Department of Urology, The First Affiliated Hospital of Jinan University, 613 West Hangpu Road, Guangzhou, Guangdong 510630, P.R. China

E-mail: zexuan_sugz@163.com

*Contributed equally

Key words: eupatilin, renal cell carcinoma, reactive oxygen species, apoptosis

\section{Introduction}

Renal cell carcinoma (RCC), accounting for $\sim 3 \%$ of all malignancies, consists of a wide range of malignancies of various histological subtypes that arise from the renal parenchyma (1). The incidence of RCC is increasing by a rate of $\sim 2.5 \%$ each year (2). Despite recent improvements in surgical and anticancer drugs, the prognosis of RCC remains poor. This is largely attributed to a lack of complete understanding of the exact underlying mechanisms that lead to this malignancy (3). Therefore, there is an urgent need to improve our understanding of the molecular mechanisms underlying the processes responsible for the development of RCC.

Reactive oxygen species (ROS), including superoxide and hydrogen peroxide, are able to regulate cysteine-based phosphatases, for example protein tyrosine phosphatases and lipid phosphatases, and directly influence cell signaling pathways (4). ROS are one of the major causes of tumors and have a significant role in the process of tumor progression, metastasis and apoptosis. Chronic and persistent exposure to high ROS levels induces DNA, protein and lipid damage, and may lead to cellular senescence and apoptosis (5). Therefore, targeting ROS is an important therapeutic strategy for the treatment of cancer.

Artemisia asiatica has been utilized in traditional Chinese medicine for centuries (6). Eupatilin (5,7-dihydroxy-3',4', 6-trimethoxyflavone) is one of the pharmacologically active components found in A. asiatica (7). Previous studies have reported that it has a variety of biological properties, including immune regulation, anti-inflammatory, anti-ulcer and anti-oxidative activity (8-11). Furthermore, there is increasing evidence that eupatilin possesses anti-tumor effects. It has been demonstrated that eupatilin inhibits the growth of human endometrial cancer cells through $\mathrm{G} 2 / \mathrm{M}$ phase cell cycle arrest via the upregulation of p21 (12). In addition, eupatilin also inhibited human gastric cancer cell growth in a doseand time-dependent manner, and induced apoptosis with a concomitant increase in caspase-3 activity (7).

However, the role of eupatilin in RCC remains to be elucidated. Therefore, the present study investigated the biological effects and mechanisms underlying eupatilin action in RCC 
cell apoptosis. In the present study, it was demonstrated that eupatilin significantly induces renal cancer cell apoptosis. The mechanisms of eupatilin's action included ROS accumulation, mitogen activated protein kinase (MAPK) activation and phosphatidylinositol 3-kinase (PI3K)/AKT activation.

\section{Materials and methods}

Cell culture and reagents. The 786-O human $\mathrm{RCC}$ cell line was purchased from American Type Culture Collection (Manassas, VA, USA). The cells were cultured in Dulbecco's modified Eagle's medium (DMEM; Gibco; Thermo Fisher Scientific, Inc., Waltham, MA, USA) containing $10 \%$ fetal bovine serum (FBS; Gibco; Thermo Fisher Scientific, Inc.) and $1 \%(\mathrm{v} / \mathrm{v})$ penicillin-streptomycin (Sigma-Aldrich, St. Louis, $\mathrm{MO}, \mathrm{USA}$ ) at $37^{\circ} \mathrm{C}$ in a humidified atmosphere containing $5 \%$ $\mathrm{CO}_{2}$. Eupatilin was obtained from YuanYe Biotechnology Co., Ltd., Shanghai, China.

Cell viability assay. Cell viability was assessed by cell counting kit (CCK)-8 assay (Beyotime Institute of Biotechnology, Haimen, China). Briefly, cells were seeded into 96-well plates at $5 \times 10^{3}$ cells per well and cultured for $24 \mathrm{~h}$ at $37^{\circ} \mathrm{C}$ to adhere. Following treatment with various concentrations of eupatilin $(10,20$ and $40 \mu \mathrm{M})$ for $72 \mathrm{~h}, 10 \mu \mathrm{l}$ of CCK-8 reagent was added to the cells, followed by incubation for $2 \mathrm{~h}$ at $37^{\circ} \mathrm{C}$. Subsequently, the optical density (OD) value was read at $450 \mathrm{~nm}$ using a Bio-Rad ELISA microplate reader (Bio-Rad Laboratories, Inc., Hercules, CA, USA). The viability rate of cells $=($ the OD values of treated groups / the OD values of the control group) $\mathrm{x} 100 \%$.

Nucleosome enzyme-linked immunosorbent assay (ELISA) assay for detection of apoptosis. For apoptosis assays, 786-O cells at a density of $1 \times 10^{4}$ cells/well were seeded into 96-well plates at $37^{\circ} \mathrm{C}$ overnight and treated with $40 \mu \mathrm{M}$ eupatilin with or withour N-acetyl-L-cysteine (NAC; $500 \mu \mathrm{M}$; Sigma-Aldrich) for $24 \mathrm{~h}$. Cells were subsequently harvested and treated with a Nucleosome ELISA kit (catalog no., QIA25-1EA; Merck Millipore, Darmstadt, Germany), according to the manufacturer's protocol.

Caspase-3 activity assay. Caspase-3 activity was determined using Caspase 3/caspase 7 Luminescent Assay kit (Invitrogen; Thermo Fisher Scientific, Inc.), according to the manufacturer's protocol. In brief, cells were lysed using RIPA assay lysis buffer (Takara Biotechnology Co., Ltd., Dalian, China), and proteins $(20 \mu \mathrm{g})$ were incubated with caspase-3 substrate DEVD-AFC $(50 \mu \mathrm{g})$ at $37^{\circ} \mathrm{C}$ for $1-2 \mathrm{~h}$. Samples were transferred into black bottom 96-well microplates and read using a fluorescence plate reader (EMD Millipore, Billerica, MA, USA). The non-cleaved (blue) and cleaved (green) substrate emissions were 400 and $505 \mathrm{~nm}$, respectively. Control reactions were performed without protein in wells and by omitting the substrate.

Measurement of cellular ROS. Intracellular ROS was measured by flow cytometry using a Active Oxygen Species Assay kit (catalog no., K0111; Beyotime Institute of Biotechnology). In brief, 786-O cells were incubated with various concentrations of eupatilin $(10,20$ and $40 \mu \mathrm{M})$ with or without NAC $(500 \mu \mathrm{M})$ for $0.5,1,2$ or $4 \mathrm{~h}$. Subsequently, the cells were washed twice with phosphate-buffered saline (PBS), and incubated with $10 \mu \mathrm{M}$ dichlorofluorescein diacetate for $30 \mathrm{~min}$ at $37^{\circ} \mathrm{C}$. The cells were subsequently trypsinized and analyzed by the FACSCaliber flow cytometer (BD Biosciences, Franklin Lakes, NJ, USA). Intracellular ROS levels were expressed as the mean dichlorofluorescein fluorescence intensity of the cells.

Measurement of glutathione(GSH)/oxidized glutathione(GSSG) ratio. Total GSH was measured using 5,5-dithio-bis-(2-nitrobenzoic acid) (DTNB) according to a previously described method (13). In brief, 786-O cells were cultured with or without various concentrations of eupatilin $(10,20$ and $40 \mu \mathrm{M})$ for $24 \mathrm{~h}$ at room temperature. Subsequently, the cells were treated with 5\% 5-sulfosalicylic acid for $30 \mathrm{sec}$. Following centrifugation (6,000 x g for $10 \mathrm{~min}$ at room temperature), the resultant extract was assayed, and the GSSG concentration was obtained by quantifying the reduction of DTNB due to its conversion to 5-thio-2-nitrobenzoic acid at $412 \mathrm{~nm}$.

Western blot analysis. Proteins were collected from 786-O cells treated with various concentrations of eupatilin (10, 20 and $40 \mu \mathrm{M})$. Cells were washed in PBS and lysed using RIPA assay lysis buffer. The concentration of protein was measured by bicinchoninic acid assay kit (Invitrogen; Thermo Fisher Scientific, Inc.), according to the manufacturer's protocol. Protein samples $(40 \mu \mathrm{g})$ were subjected to $12 \%$ sodium dodecyl sulfate-polyacrylamide gel electrophoresis and transferred to polyvinylidene difluoride (PVDF) membranes (EMD Millipore). PVDF membranes were blocked with $5 \%$ bovine serum albumin (Sigma-Aldrich) in PBS with tween, followed by incubation with primary antibody at $4^{\circ} \mathrm{C}$ overnight. Detection of p38 (1:1,500; catalog no., sc-81621), phosphorylated (p)-p38 (1:1,500; catalog no., sc-7973), c Jun N terminal kinases (JNK; 1:1,000; catalog no., sc-7345), p-JNK (1:1,000; catalog no., sc-6254), extracellular signal-regulated kinase 1/2 (ERK; 1:1,500; catalog no., sc-514302), p-ERK (1:1,500; catalog no., sc-7383), PI3K (1:2,000; catalog no., sc-365290), AKT (1:2,000; catalog no., sc-5298) and $\beta$-actin $(1: 1,500$; catalog no., sc-21733) was performed using mouse monoclonal antibodies from Santa Cruz Biotechnology, Inc. (Dallas, TX, USA). p-PI3K (1:2,000; catalog no., sc-293115) and p-AKT (1:2,000; catalog no., sc-135650) were detected using rabbit monoclonal and rabbit polyclonal antibodies, respectively, (Santa Cruz Biotechnology, Inc.). Subsequently, the blots were washed with TBST and incubated with bovine anti-rabbit and rabbit anti-mouse horseradish peroxidase-conjugated secondary antibodies $(1: 3,000$; catalog nos., sc-2379 and sc-358914, respectively, Santa Cruz Biotechnology, Inc.) at room temperature for $2 \mathrm{~h}$. An enhanced chemiluminescence system was applied to visualize the blots according to the manufacturer's protocol (Roche Applied Science, Mannheim, Germany). The relative protein expression levels were quantified using Image-Pro Plus version 6.0 software (Media Cybernetics, Silver Spring, MD, USA) and normalized to $\beta$-actin.

Statistical analysis. Results are expressed as the mean \pm standard deviation. Comparisons between two groups were 


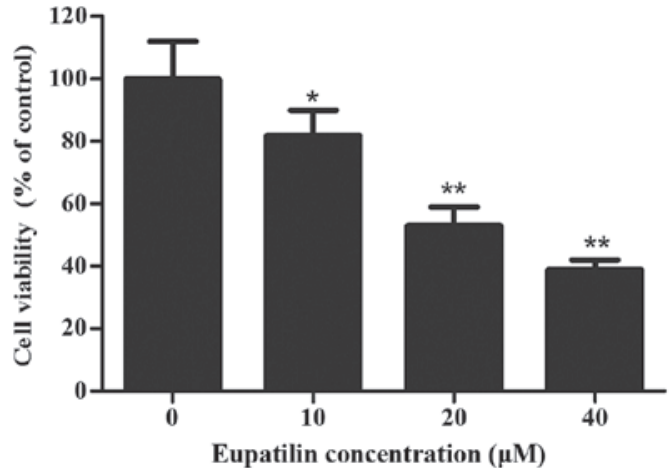

Figure 1. Effects of eupatilin treatment on cell viability in 786-O cells. $786-\mathrm{O}$ cells $\left(1 \times 10^{4} /\right.$ well $)$ in 96 -well plates were pretreated with various concentrations of eupatilin $(10,20$ and $40 \mu \mathrm{M})$ for $72 \mathrm{~h}$, and the cell counting kit- 8 assay was performed to detect cell viability. Eupatilin significantly suppressed the viability of $786-O$ cells. Data represent the mean \pm standard deviation of three independent experiments. " $\mathrm{P}<0.05$ compared with the control group; ${ }^{* *} \mathrm{P}<0.01$ compared with the control group.

performed using the Student's t-test and between multiple groups using analysis of variance. SPSS version 13.0 software (SPSS, Inc., Chicago, IL, USA) was used for statistical analysis. $\mathrm{P}<0.05$ was considered to indicate a statistically significant difference.

\section{Results}

Eupatilin inhibits proliferation in 786-O cells. To investigate the cytotoxicity of eupatilin, 786-O cells were treated with various concentrations of eupatilin for $72 \mathrm{~h}$, followed by CCK-8 assay. As shown in Fig. 1, eupatilin significantly inhibited cell viability in a concentration-dependent manner $(\mathrm{P}<0.05)$, and the cell viability of 20 and $40 \mu \mathrm{M}$ eupatilin-treated 786-O cells was decreased by 47.2 and $61.3 \%$, respectively. Due to the prominent proliferation inhibition of $786-\mathrm{O}$ cells, $40 \mu \mathrm{M}$ of eupatilin was used for the majority of the subsequent assays.

Eupatilin induces apoptosis in 786-O cells. Subsequently. Nucleosome ELISA assays were performed to measure the effect of eupatilin treatment on cell apoptosis. As shown in Fig. 2A, the eupatilin treatment group had an increased number of apoptotic cells compared with the control group $(\mathrm{P}<0.05)$. In addition, the caspase- 3 activity was determined in the various groups. The activity of caspase- 3 was markedly increased in the eupatilin treatment group compared with the control group (Fig. 2B; P<0.05). The results of the present study indicated that eupatilin induced apoptosis in 786-O cells.

Eupatilin induces oxidative stress in 786-O cells. ROS have a significant role in the processes of tumor progression, metastasis and apoptosis (14). Therefore, the present study investigated the effect of eupatilin treatment on ROS production in 786-O cells. When 786-O cells were exposed to eupatilin for $24 \mathrm{~h}$, eupatilin significantly increased the levels of ROS in 786-O cells compared with the control group (Fig. 3A; $\mathrm{P}<0.05$ ). In addition, the GSH/GSSG ratio was markedly decreased by eupatilin (Fig. 3B). The antioxidant
A

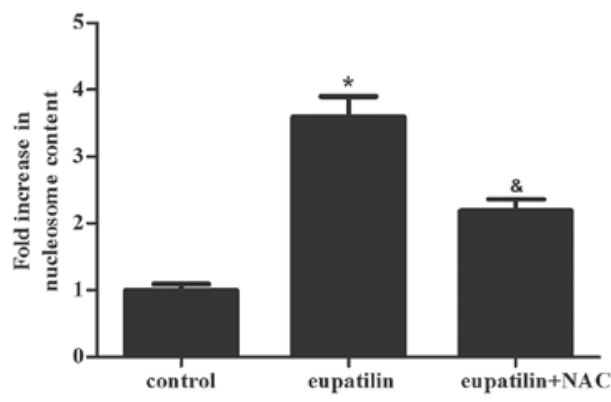

B

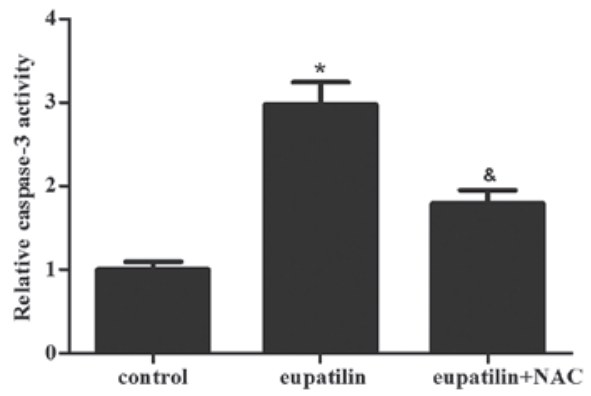

Figure 2. Effects of eupatilin treatment on apoptosis in 786-O cells. The cells were treated with $40 \mu \mathrm{M}$ eupatilin with or without NAC $(500 \mu \mathrm{M})$ for 24 h. (A) Cell apoptosis was performed using a Nucleosome ELISA kit. (B) Caspase-Glo ${ }^{\circledR} 3 / 7$ assay was used to measure the activity of caspase-3. Data are presented as the mean \pm standard deviation of three independent experiments. ${ }^{*} \mathrm{P}<0.05$ compared with the control group; ${ }^{\&} \mathrm{P}<0.05$ compared with the eupatilin alone group $(40 \mu \mathrm{M})$. NAC, N-acetyl-L-cysteine.
A

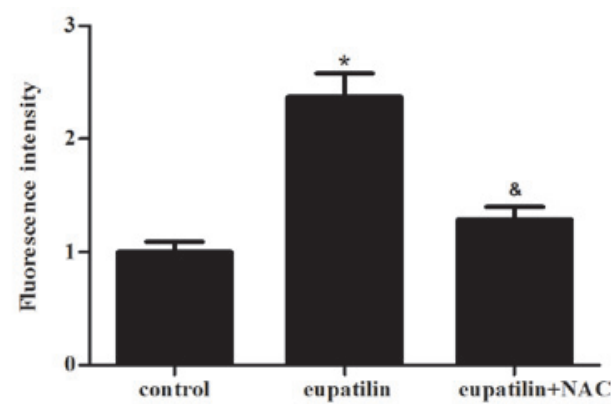

B

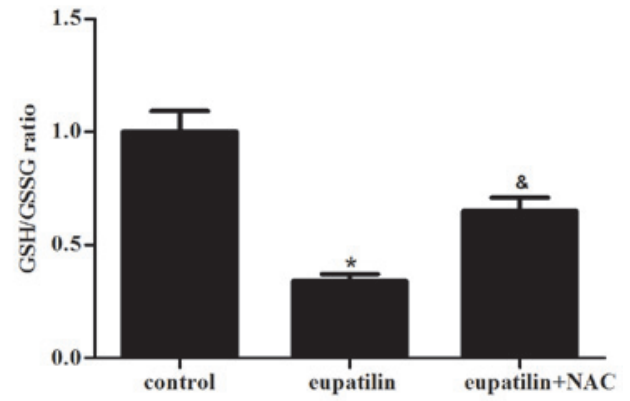

Figure 3. Effects of eupatilin treatment on redox state in 786-O cells. The cells were treated with $40 \mu \mathrm{M}$ eupatilin with or without NAC $(500 \mu \mathrm{M})$ for 24 h. (A) The dichlorofluorescein diacetate was used to determine reactive oxygen species level. (B) Oxidative stress was measured by GSH/GSSG ratio. Data are presented as the mean \pm standard deviation of three independent experiments. ${ }^{*} \mathrm{P}<0.05$ compared with the control group; ${ }^{\&} \mathrm{P}<0.05$ compared with the eupatilin alone group $(40 \mu \mathrm{M})$. NAC, N-acetyl-L-cysteine; GSH, reduced glutathione; GSSG, oxidized glutathione.

NAC $(500 \mu \mathrm{M})$, a precursor of GSH, effectively prevented eupatilin-induced ROS production and GSH/GSSG ratio reduction (Fig. 3A and B). 


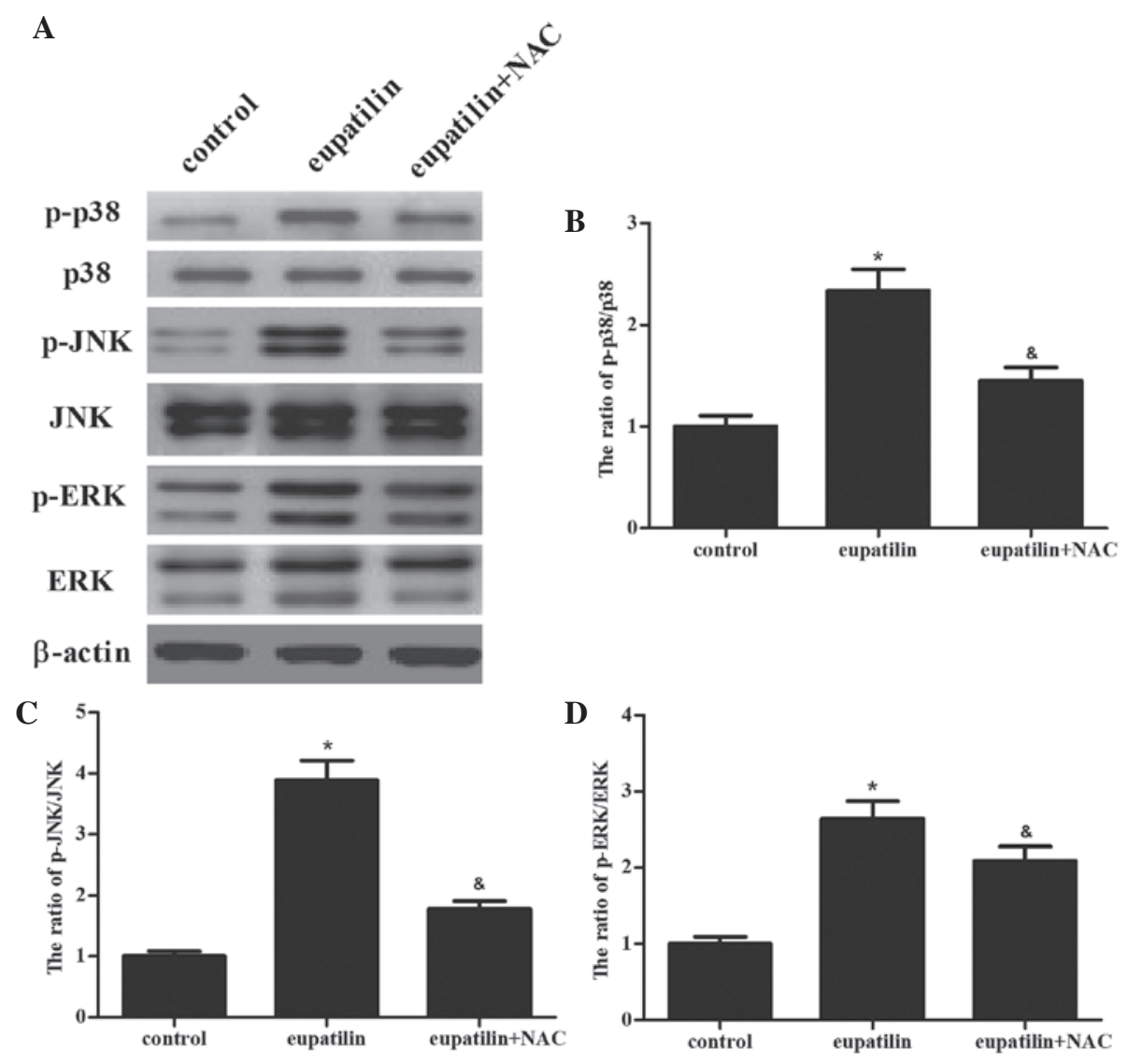

Figure 4. Eupatilin treatment activates mitogen-activated protein kinase signaling pathways in 786-O cells. The cells were treated with $40 \mu \mathrm{M}$ eupatilin with or without NAC $(500 \mu \mathrm{M})$ for $24 \mathrm{~h}$. (A) Equal amounts (30 $\mu \mathrm{g}$ protein per lane) of total proteins were separated by sodium dodecyl sulfate-polyacrylamide gel electrophoresis and analyzed by immunoblotting with anti-p-p38, anti-p38, anti-p-ERK, anti-ERK, anti-p-JNK and anti-JNK antibodies. (B-D) Quantitative analysis of p-p38, p-JNK and p-ERK protein levels. Data are presented as the mean \pm standard deviation of three independent experiments. " $\mathrm{P}<0.05$ compared with the control group; ${ }^{\circledR} \mathrm{P}<0.05$ compared with the eupatilin alone group $(40 \mu \mathrm{M})$. NAC, N-acetyl-L-cysteine; p, phosphorylated; ERK, extracellular signal-regulated kinase; JNK, c-Jun N-terminal kinase.

Eupatilin activates ERK, JNK and p38 in 786-O cells. ROS have been demonstrated to be an inducer or mediator of the activation of mitogen-activated protein kinase (MAPK) family members, including JNK, p38 and ERK1/2 (15). In addition, the MAPK signaling pathway has a significant role in the regulation of a number of cellular processes, including cell growth and proliferation, differentiation, and apoptosis (16). Therefore, the present study investigated whether eupatilin was able to induce the activation of MAPK cascades in 786-O cells. As shown in Fig. 4, it was observed that eupatilin induced phosphorylation of $\mathrm{p} 38 \alpha$ (Thr180/Tyr182), ERK1/2 and JNK1/2 (Thr183/Tyr185) in 786-O cells. Subsequently, the role of ROS in eupatilin-mediated ERK, JNK, and p38 MAPK activation was investigated in 786-O cells. The results of the present study demonstrated that pretreatment with NAC clearly prevented the activation of the protein kinases induced by eupatilin treatment.

Eupatilin suppresses the PIBK/AKT signaling cascade in 786-O cells. Besides MAPK signaling pathways, the PI3K/AKT signal transduction pathway additionally has a critical role in cell survival and the enhanced protection of cancer cells from apoptosis during tumorigenesis (17). The present study investigated whether eupatilin downregulated PI3K/AKT activation in 786-O cells. Western blotting demonstrated that eupatilin significantly suppressed the activation of PI3K and AKT. Furthermore, pretreatment with NAC reversed the eupatilin-inhibited activation of PI3K and AKT in 786-O cells (Fig. 5; $\mathrm{P}<0.05$ ). The results of the present study indicated that the inhibition of the PI3K/AKT signaling cascade by eupatilin led to the suppression of cell proliferation in 786-O cells.

\section{Discussion}

Previous studies have demonstrated that eupatilin inhibits cell proliferation, migration and invasion, and promotes apoptosis in various cancer cells $(7,12)$. In the present study, it was demonstrated that eupatilin treatment significantly induced 786-O cell apoptosis. The anticancer effect of eupatilin depends on enhanced ROS in 786-O cells. ROS-dependent MAPK activation and inhibition of PI3K/AKT contribute to eupatilin's effects on RCC cell apoptosis.

ROS are primarily produced in mitochondria as a product of normal cellular metabolism of oxygen, and function as significant molecules for mediating normal physiological signaling (18). In addition, elevated ROS production followed by oxidative stress is involved in the initiation and promotion of multistep carcinogenesis (19). Apoptosis has been recognized as one of the most important biological features 

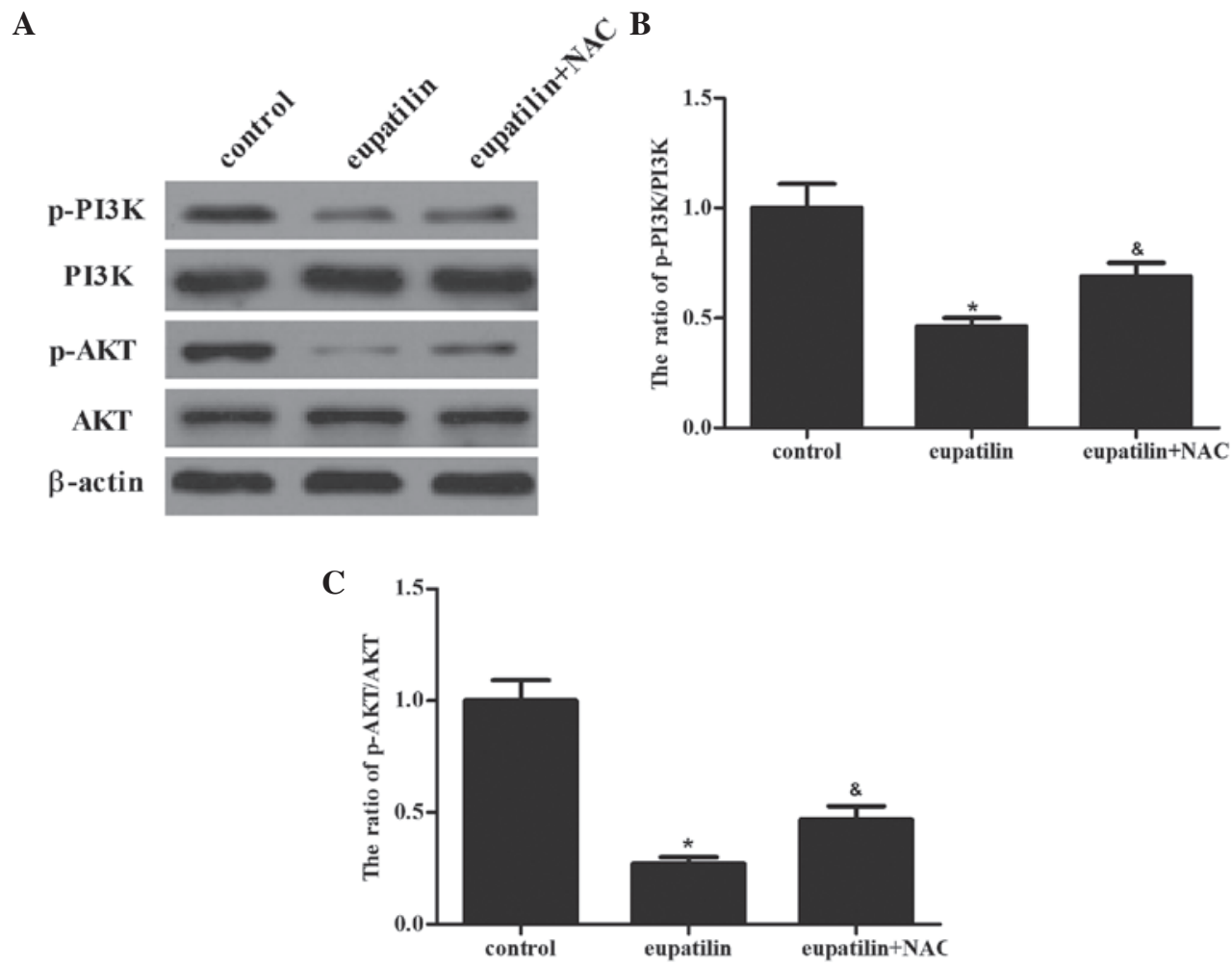

Figure 5. Eupatilin treatment suppresses PI3K/AKT signaling pathway in 786-O cells. The cells were treated with $40 \mu \mathrm{M}$ eupatilin with or without NAC $(500 \mu \mathrm{M})$ for $24 \mathrm{~h}$. (A) Equal amounts (30 $\mu \mathrm{g}$ protein per lane) of total proteins were separated by sodium dodecyl sulfate-polyacrylamide gel electrophoresis and analyzed by immunoblotting with anti-p-PI3K, anti-PI3K, anti-p-AKT and anti-AKT antibodies. (B and C) Quantitative analysis of p-PI3K and p-AKT protein levels. Data are presented as the mean \pm standard deviation of three independent experiments. ${ }^{*} \mathrm{P}<0.05$ compared with the control group; ${ }^{\&} \mathrm{P}<0.05$ compared with the eupatilin alone group $(40 \mu \mathrm{M})$. PI3K, phosphoinositide 3-kinase; NAC, N-acetyl-L-cysteine; p, phosphorylated.

of RCC progression, and the execution of apoptosis requires the accurate coordination of the caspase protein family (20). Caspase-3, also known as the death enzyme, plays a critical role in the controlled execution of programmed cell death (21). The results of the present study reveal that eupatilin significantly inhibited RCC cell proliferation, and induced RCC apoptosis, as well as the activity of caspase-3. In addition, eupatilin treatment dramatically induced the production of ROS and decreased the ratio of GSH/GSSG in 786-O cells. The present study subsequently investigated whether ROS was involved in eupatilin-induced apoptosis. As expected, pretreatment with NAC clearly prevented eupatilin-induced apoptosis, as evidenced by decreased caspase- 3 cleavage. The results of the present study suggested that ROS has a significant role in eupatilin-induced apoptosis in RCC cells.

MAPKs, including ERK1/2, JNK and p38, are a family of serine/threonine kinases that regulate a variety of cellular events, including proliferation and apoptosis $(16,22,23)$. A previous study demonstrated that various stress stimuli, including the oxidative stress caused by ROS, may induce potential activation of MAPK signaling pathways (24). In addition, the activation of JNK contributes to stress-induced apoptosis (25). p38 MAPK has been demonstrated to either promote apoptosis or enhance cell survival depending on the cell type and stimulus $(26,27)$. The PI3K/AKT signaling pathway is one of the major signaling pathways associated with RCC progression (28-30). It is involved in propagation of signals from various cell membrane receptor tyrosine kinases into the nucleus, and regulates various cellular processes, including cell proliferation, differentiation and apoptosis (31). In numerous cancer tissues and cells, the PI3K/AKT signaling pathway is overactive, reducing apoptosis and allowing proliferation (32). ROS have been demonstrated to be associated with apoptosis induced by anticancer drugs and may be upstream of MAPK and PI3K/AKT signaling pathways (33). Hao et al (34) reported that licochalcone A, a flavonoid extracted from licorice root, inhibited cell proliferation and induced gastric cell apoptosis via modulation of ROS-mediated MAPKs and PI3K/AKT signaling pathways. The results of the present study demonstrate that eupatilin induces phosphorylation of p38 $\alpha$ (Thr180/Tyr182), ERK1/2 and JNK1/2 (Thr183/Tyr185), and decreases the phosphorylation of PI3K and AKT in 786-O cells in a concentration-dependent manner. It was also observed that the ROS inhibitor NAC was able to rescue the MAPK activation, PI3K/AKT inhibition and apoptosis induced by inhibition of eupatilin. The results of the present study suggest that eupatilin induces ROS-mediated MAPK activation, inhibits the PI3K/AKT signaling pathway and leads to RCC cell apoptosis.

In conclusion, the results of the present study provide evidence that inhibition of eupatilin induces apoptosis in human RCC through ROS-mediated activation of the MAPK signaling pathway and inhibition of the PI3K/AKT signaling pathway. Therefore, eupatilin may serve as a potential therapeutic agent for the treatment of human RCC. Xenograft nude mice may be employed in the future to examine the role of eupatilin in tumorigenesis of RCC. 


\section{References}

1. Larkin J, Goh XY, Vetter M, Pickering L and Swanton C: Epigenetic regulation in RCC: Opportunities for therapeutic intervention? Nat Rev Urol 9: 147-155, 2012.

2. Jemal A, Siegel R, Xu J and Ward E: Cancer statistics, 2010. CA Cancer J Clin 60: 277-300, 2010.

3. Penticuff JC and Kyprianou N: Therapeutic challenges in renal cell carcimoma. Am J Clin Exp Urol 3: 77-90, 2015.

4. Salmeen A and Barford D: Functions and mechanisms of redox regulation of cysteine-based phosphatases. Antioxid Redox Signal 7: 560-577, 2005.

5. Waris G and Ahsan H: Reactive oxygen species: Role in the development of cancer and various chronic conditions. J Carcinog 5: 14, 2006.

6. Jang HJ, Jeong EK, Kim SS, Lee JH, Oh MY, Kang KS, Kwan HC, Song KI, Eom DW and Han DJ: Protective effect of Artemisia asiatica extract against renal ischemia-reperfusion injury in mice. Exp Clin Transplant Suppl 1: 377-82, 2015.

7. Park BB, Yoon JS, Kim ES, Choi J, Won YW, Choi JH and Lee YY: Inhibitory effects of eupatilin on tumor invasion of human gastric cancer MKN-1 cells. Tumor Biol 34: 875-885, 2013.

8. Kim YD, Choi SC, Oh TY, Chun JS and Jun CD: Eupatilin inhibits T-cell activation by modulation of intracellular calcium flux and NF-kappaB and NF-AT activity. J Cell Biochem 108: 225-236, 2009.

9. Choi EJ, Lee S, Chae JR, Lee HS, Jun CD and Kim SH: Eupatilin inhibits lipopolysaccharide-induced expression of inflammatory mediators in macrophages. Life Sci 88: 1121-1126, 2011.

10. Yoon KD, Chin YW, Yang MH and Kim J: Separation of anti-ulcer flavonoids from Artemisia extracts by high-speed countercurrent chromatography. Food Chem 129: 679-683, 2011.

11. Choi EJ, Oh HM, Na BR, Ramesh TP, Lee HJ, Choi CS, Choi SC, Oh TY, Choi SJ, Chae JR, et al: Eupatilin protects gastric epithelial cells from oxidative damage and down-regulates genes responsible for the cellular oxidative stress. Pharm Res 25: 1355-1364, 2008.

12. Cho JH, Lee JG, Yang YI, Kim JH, Ahn JH, Baek NI, Lee KT and Choi JH: Eupatilin, a dietary flavonoid, induces G2/M cell cycle arrest in human endometrial cancer cells. Food Chem Toxicol 49: 1737-1744, 2011.

13. Jin Y, Yang J, Lin L, Lin Y and Zheng C: The attenuation of Scutellariae radix extract on oxidative stress for colon injury in lipopolysaccharide-induced RAW264.7 cell and 2,4,6-trinitrobenzene sulfonic acid-induced ulcerative colitis rats. Pharmacogn Mag 12: 153-159, 2016.

14. Xiong Y, Ye T, Wang M, Xia Y, Wang N, Song X, Wang F, Liu L, Zhu Y, Yang F, et al: A novel cinnamide YLT26 induces breast cancer cells apoptosis via ROS-mitochondrial apoptotic pathway in vitro and inhibits lung metastasis in vivo. Cell Physio Biochem 34: 1863-1876, 2014.

15. Choi EK, Yeo JS, Park CY, Na HI, Lim JA, Lee JE, Hong SW, Park SS, Lim DG and Kwak KH: Inhibition of reactive oxygen species downregulates the MAPK pathway in rat spinal cord after limb ischemia reperfusion injury. Int J Surg 22: 74-81, 2015.

16. Huang D, Ding Y, Luo WM, Bender S, Qian CN, Kort E, Zhang ZF, VandenBeldt K, Duesbery NS, Resau JH and Teh BT: Inhibition of MAPK kinase signaling pathways suppressed renal cell carcinoma growth and angiogenesis in vivo. Cancer Res 68: 81-88, 2008.

17. Guo H, German P, Bai S, Barnes S, Guo W, Qi X, Lou H, Liang J, Jonasch E, Mills GB and Ding Z: The PI3K/AKT pathway and renal cell carcinoma. J Genet Genomics 42: 343-353, 2015.
18. Thannickal VJ and Fanburg BL: Reactive oxygen species in cell signaling. Am J Physiol Lung Cell Mol Physiol 279: L1005-L1028, 2000.

19. Essick EE and Sam F: Oxidative stress and autophagy in cardiac disease, neurological disorders, aging and cancer. Oxid Med Cell Longev 3: 168-177, 2010.

20. Li J and Yuan J: Caspases in apoptosis and beyond. Oncogene 27: 6194-6206, 2008.

21. Tawa P, Hell K, Giroux A, Grimm E, Han Y, Nicholson DW and Xanthoudakis S: Catalytic activity of caspase-3 is required for its degradation: Stabilization of the active complex by synthetic inhibitors. Cell Death Differ 11: 439-447, 2004.

22. Wada T and Penninger JM: Mitogen-activated protein kinases in apoptosis regulation. Oncogene 23: 2838-2849, 2004.

23. Ballif BA and Blenis J: Molecular mechanisms mediating mammalian mitogen-activated protein kinase (MAPK) kinase (MEK)-MAPK cell survival signals. Cell Growth Differ 12: 397-408, 2001

24. Liu J, Chang F, Li F, Fu H, Wang J, Zhang S, Zhao J and Yin D: Palmitate promotes autophagy and apoptosis through ROS-dependent JNK and p38 MAPK. Biochem Biophys Res Commun 463: 262-267, 2015.

25. Ki YW, Park JH, Lee JE, Shin IC and Koh HC: JNK and p38 MAPK regulate oxidative stress and the inflammatory response in chlorpyrifos-induced apoptosis. Toxicol Lett 218: 235-245, 2013.

26. Porras A, Zuluaga S, Black E, Valladares A, Alvarez AM, Ambrosino C, Benito $M$ and Nebreda AR: P38 alpha mitogen-activated protein kinase sensitizes cells to apoptosis induced by different stimuli. Mol Biol Cell 15: 922-933, 2004.

27. Park EJ, Park SW, Kim HJ, Kwak JH, Lee DU and Chang KC: Dehydrocostuslactone inhibits LPS-induced inflammation by p38MAPK-dependent induction of hemeoxygenase-1 in vitro and improves survival of mice in CLP-induced sepsis in vivo. Int Immunopharmacol 22: 332-340, 2014

28. Porta C and Figlin RA: Phosphatidylinositol-3-kinase/Akt signaling pathway and kidney cancer, and the therapeutic potential of phosphatidylinositol-3-kinase/Akt inhibitors. J Urol 182: 2569-2577, 2009.

29. Banumathy G and Cairns P: Signaling pathways in renal cell carcinoma. Cancer Biol Ther 10: 658-664, 2010.

30. Sourbier C, Lindner V, Lang H, Agouni A, Schordan E, Danilin S, Rothhut S, Jacqmin D, Helwig JJ and Massfelder T: The phosphoinositide 3-kinase/Akt pathway: A new target in human renal cell carcinoma therapy. Cancer Res 66: 5130-5142, 2006.

31. Brzezianska E and Pastuszak-Lewandoska D: A minireview: The role of MAPK/ERK and PI3K/Akt pathways in thyroid follicular cell-derived neoplasm. Fronti Biosci (Landmark Ed) 16: 422-439, 2011.

32. Shi Y, Song Q, Hu D, Zhuang X, Yu S and Teng D: Oleanolic acid induced autophagic cell death in hepatocellular carcinoma cells via PI3K/Akt/mTOR and ROS-dependent pathway. Korean J Physiol Pharmacol 20: 237-243, 2016.

33. Mi Y, Xiao C, Du Q, Wu W, Qi G and Liu X: Momordin Ic couples apoptosis with autophagy in human hepatoblastoma cancer cells by reactive oxygen species (ROS)-mediated PI3K/Akt and MAPK signaling pathways. Free Radic Biol Med 90: 230-422, 2016.

34. Hao W, Yuan X, Yu L, Gao C, Sun X, Wang D and Zheng Q: Licochalcone A-induced human gastric cancer BGC-823 cells apoptosis by regulating ROS-mediated MAPKs and PI3K/AKT signaling pathways. Sci Rep 5: 10336, 2015. 